\title{
3D Printed Periodic Structures for Subterahertz Sources
}

\author{
D. I. Sobolev, M.D. Proyavin, N.Yu. Peskov, V.Yu. Zaslavsky, V.V. Parshin \\ Institute of Applied Physics, Nizhny Novgorod, Russia, nrtnm@mail.ru
}

Recent advances in 3D printing technology made it a favorable choice in many scientific applications, where large-scale production is not required. There are several different materials and printing methods available, which are more or less suitable for subterahertz devices. Selective laser sintering (SLS) printers can reach tolerances down to several microns, although these are on the expensive side, and in the case of metallic printing the surface roughness is unsatisfactory for radiation with millimeter and submillimeter wavelengths without additional finishing. Moreover, SLS printing of copper is very difficult due to its high reflection coefficient at commonly used laser wavelengths. Stereolithography (SLA) photopolymer printers have very high accuracy, but these plastics usually have high absorption in microwave and terahertz region. Fused-deposition modelling (FDM) printers are cheap, widely available and can use several well-known plastics with low absorption, such as nylon or HDPE, but the precision is less than for the former types.

To study the best possible solutions, we analyzed the dielectric permittivity and loss tangent of plastics used in different 3D printer types. Sample discs were printed and then placed inside the two-mirror resonator with high $\mathrm{Q}$ factor [1], and measurements were made for frequencies in $94-150 \mathrm{GHz}$ range. Additional measurements were made for plastic waveguide inserts in 26-40 $\mathrm{GHz}$ range. Dielectric properties in different ranges have only a slight difference and can therefore be extrapolated to higher frequencies. Measurement results are collected in the table.

\begin{tabular}{|l|l|l|l|}
\hline Plastic & Printer & $\operatorname{Re} \varepsilon$ & $\tan \delta$ \\
\hline $\begin{array}{l}\text { Polyethylene terephthalate } \\
\text { glycol (PETG) } 1\end{array}$ & FDM & 2.31 & $1.5 \cdot 10^{-3}$ \\
\hline PETG 2 & FDM & 2.47 & $1.6 \cdot 10^{-3}$ \\
\hline Polylactic Acid (PLA) & FDM & 2.27 & $6.2 \cdot 10^{-3}$ \\
\hline Sterol-butadiene-sterol (SBS) 1 & FDM & 2.22 & $1.6 \cdot 10^{-3}$ \\
\hline SBS 2 & FDM & 2.40 & $1.3 \cdot 10^{-3}$ \\
\hline Visijet SL Clear & SLA & 1.8 & $3.5 \cdot 10^{-2}$ \\
\hline Visijet SL Flex & SLA & 1.8 & $3.5 \cdot 10^{-2}$ \\
\hline Visijet SL Hi-Temp & SLA & 1.8 & $3.5 \cdot 10^{-2}$ \\
\hline Polyamid & SLS & 1.7 & $3.5 \cdot 10^{-3}$ \\
\hline & \multicolumn{3}{|l}{}
\end{tabular}

Sterol-Butadiene-Sterol has the best dielectric properties among the measured samples, and it was chosen to test the concept of two-dimensional planar Bragg resonator with chessboard-like grating, which can be used in distributed feedback lasers [2]. The parameters of Bragg structure were chosen in accordance with dielectric properties of the media and precision of FDM printers, which is acceptable for frequencies up to $100 \mathrm{GHz}$. Size of the square resonator is $180 \mathrm{~mm}$, and average thickness is $2 \mathrm{~mm}$. Grating periods in orthogonal directions are both equal to 4 $\mathrm{mm}$, and grating depth is $0.4 \mathrm{~mm}$. The 3D-printed structure is shown on Fig. 1.
The properties of this resonator were calculated in CST MWS for $\operatorname{Re} \varepsilon=2.4$ and $\tan \delta=2 \cdot 10^{-3}$, and the zone of effective scattering was near $60 \mathrm{GHz}$. To conduct the low-power measurement, the resonator had to be excited by a wave beam with a plane phase front, which was be made by a planar quasioptical transmission line. The transmission line consists of two parallel metallic planes, parabolic mirror and single-mode waveguide ending in the focus of the mirror. After the reflection, the planar wave beam is very close to the TEM-mode of planar waveguide. The wave beam is transmitted through the resonator and then received into single-mode waveguide by the symmetrical quasioptical transmission line.

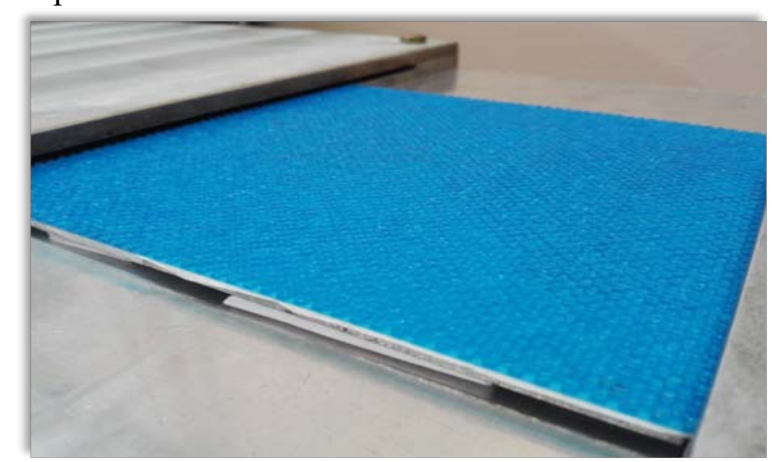

Fig. 1. Dielectric two-dimensional plane Bragg resonator printed from SBS plastic

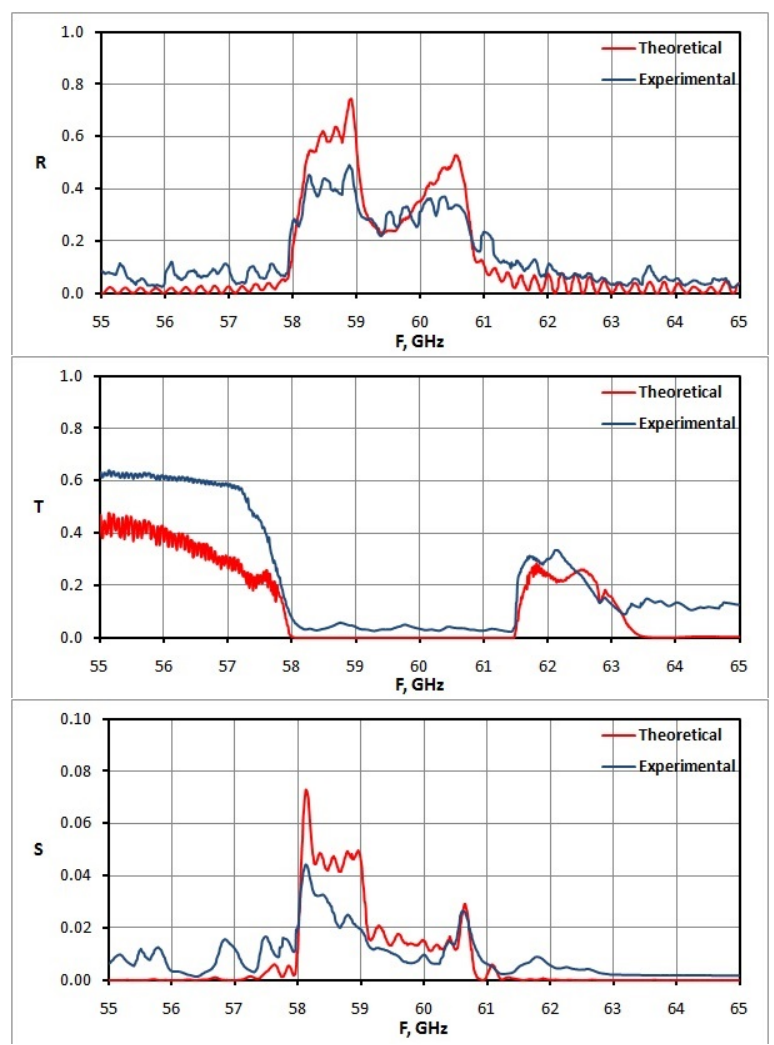

Fig. 2. Reflection (R), transmission (T) and orthogonal scattering (S) for dielectric two-dimensional plane Bragg resonator 
Measurement results for reflection, transmission and orthogonal scattering are shown on the Fig. 2. There is a good agreement between calculations and experiment for both frequencies of the effective Bragg scattering and amplitudes of the scattering coefficients. The use of higher precision printers will allow manufacture of resonators suitable for submillimeter wavelengths.

Another promising field for printed dielectric structures is low-loss windows. Calculations basing on thermal and dielectric properties of printable plastics give the power of several tens of watts, which is sufficient for many applications, e.g. subterahertz gyrotron systems used for spectroscopy [3]. Window reflection can be minimized by the use of gratings of different shapes, like two-dimensional pyramidal or one-dimensional close to sinusoidal [4]. The shape of the grating requires fine details to be printed with high precision, and single-nozzle FDM printers flatten the top parts of the shape, which leads to the increase of the reflection. SLS plastic printers have significantly better precision. To compare different gratings, three discs were printed of polyamid: the first one has no gratings on the sides, the second one has pyramids with height $2 \mathrm{~mm}$ and base width $1 \mathrm{~mm}$ on both sides, and the third one has special one-dimensional periodic grating with height $3 \mathrm{~mm}$ and period $2 \mathrm{~mm}$ on both sides. The discs are shown on Fig. 3.

The low-power tests were conducted on the same resonator setup, which was used for the tests of dielectric properties. The measurements performed at frequencies $70-200 \mathrm{GHz}$ show the significant reduction of reflection: both types of gratings effectively suppressed the reflection by more than $10 \mathrm{~dB}$. This is sufficient for many subterahertz gyrotrons, and additional tests of mechanical and vacuum properties will be performed later.

This work was supported by RSCF grant 18-48520022. Authors are grateful to DS Group for their help with manufacture of samples.

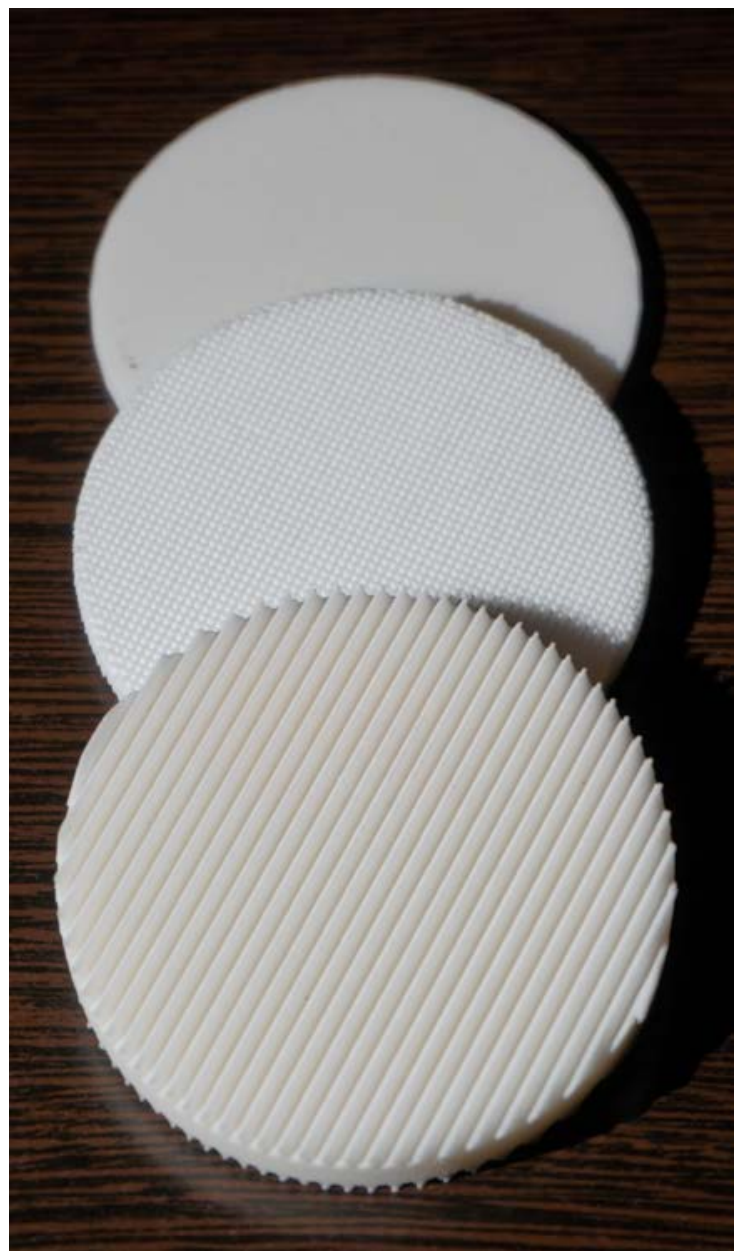

Fig. 3. Printed discs for antireflection grating tests: top - no gratings, middle - pyramidal gratings, bottom - onedimensional gratings.

\section{References}

1. Dryagin, Y.A. \& Parshin, V.V. A method to measure dielectric parameters in 5-0.5 millimeter wavelength band // Int. J. Infrared Milli. Waves. 1987. V. 13, No. 7. P. 10231032.

2. Ginzburg, N.S., Peskov, N.Yu., Sergeev, A.S. Dynamics of free-electron lasers with two-dimension distributed feedback // Optics Commun. 1994. V.112. P.151-156.

3. Glyavin, M.Yu., Idehara, T., Sabchevski, S.P. Development of THz gyrotrons at IAP RAS and FIR UF and their applications in physical research and high-power $\mathrm{THz}$ technologies // IEEE Trans. Terahertz Sci. Techn. 2015. V. 5, No. 5, P. 788-797.

4. Vlasov, S. N., Koposova, E. V. Suppressing the reflection from an interface between dielectrics by corrugating their surfaces // Techn. Phys. 1993. V. 38, No. 7. P. 592595. 